\title{
What is VAERS?
}

\begin{abstract}
VAERS is the Vaccine Adverse Events Reporting System (VAERS), which is used to collect annual reports about adverse events associated with the vaccines. The purpose is to ensure the safe usage of vaccines. As providers of immunization services, pharmacists are required to report certain vaccine-related adverse events. This article explains the key issues that pharmacists need to know when reporting vaccine adverse events as well as providing immunization services.
\end{abstract}

Keywords: VAERS, vaccine adverse events, vaccine administration
Volume I Issue I - 2015

\section{Sandra Chinazo Nwankwo}

Woodmoor Pharmacy in Baltimore, USA

Correspondence: Sandra Chinazo Nwankwo, Woodmoor Pharmacy in Baltimore, Balitimore, USA, Tel 410-262-2467, Email l.tutunji@ju.edu.jo

Received: June II, 2015 | Published: June 29, 2015
Abbreviations: NCVIA, national childhood vaccine injury act; FDA, food and drug administration; CDC, centers for disease control and prevention; RET, reportable events table.

\section{What is the vaccine adverse events reporting system (VAERS)?}

VAERS is a national reporting system mandated by the National Childhood Vaccine Injury Act (NCVIA) in 1990, and administered by the Food and Drug Administration (FDA) and the Centers for Disease Control and Prevention (CDC) ${ }^{1,2}$ Currently VAERS receives 30,000 reports annually and has collected more than 200,000 reports since $1990 .{ }^{1}$ This post marketing safety surveillance program collects reports on adverse events associated with the vaccines. VAERS scientists analyze these reports and use the information to identify new safety concerns, ensure safe strategies of vaccine use and make sure the benefits of the vaccines outweighs the risks. The VAERS database is searchable by any member of the public. The CDC WONDER database contains reports received from 1990 to the present which contains VAERS data and report details. ${ }^{3}$

\section{Why is VAERS important to pharmacists?}

Pharmacists, as the most publically accessible health-care provider, are well positioned to identify adverse vaccine reactions in their patients and to voluntarily report these events to VAERS. Pharmacists are also providers of immunization services and as a duty of this role, are required to report certain vaccine-related adverse events.

\section{What should be reported to VAERS?}

Any significant adverse events that occur after vaccine administration of adults and children, even if you are not sure whether the vaccine caused the adverse event. It does not provide medical advice or general vaccine information. ${ }^{3}$

\section{Which adverse events must be reported to VAERS?}

The NCVIA requires healthcare providers to report:

a) Adverse event listed in the package insert.

b) Adverse events listed in the Reportable Events table (RET).

The table explains vaccine-induced injuries and conditions, and the time intervals the adverse events occur after receiving the vacci- ne. The table can be found at VAERS ${ }^{4}$ website or CDC website. ${ }^{5}$ For instance, the RET shows that anaphylaxis or anaphylactic shock is an adverse event for DTap/Td vaccine, which can occur within a seven day interval after vaccination.

\section{Who should report to VAERS?}

Physicians, pharmacists, vaccine manufacturers, vaccine recipients, and providers in state immunization programs are required to report. In addition, VAERS encourages parents, guardians and vaccine recipients to seek help from their healthcare provider in submitting reports.

\section{When should the vaccine adverse events be reported?}

VAERS encourages that reports be submitted in a timely manner; however, there are no restrictions on when a report can be submitted.

\section{How do you report to VAERS?}

Online or fax or mail. Instructions are found at VARES website. ${ }^{6}$

Why should vaccine adverse events be reported?

The VAERS information is used to evaluate vaccine safety, ensure safe approaches of vaccine use, and reduce risk associated with vaccines.

\section{Preparing to report VAERS ${ }^{3}$}

VAERS reports include five categories of information:
a. Reporter
b. Patient
c. Vaccination administration
d. Adverse event \& event outcomes
e. Vaccine history, concomitant medications and prior events

\section{VAERS follow-up ${ }^{3}$}

Reporters will receive a confirmation number called an "E-number". E-numbers are used to track specific event and allow reporters to link follow-up information to the original report. 


\section{Storage and handling concerns ${ }^{7}$}

a) According to the $\mathrm{CDC}$ guidelines, the following vaccines must be frozen $\left(-58^{\circ} \mathrm{F}\right.$ and $+5^{\circ} \mathrm{F}\left(-50^{\circ} \mathrm{C}\right.$ and $\left.\left.-15^{\circ} \mathrm{C}\right)\right)$ : Varivax, Zostavax, MMRV and MMR vaccines. Keep diluents in the refrigerator. The following vaccines must be refrigerated $\left(35^{\circ} \mathrm{F}\right.$ and $46^{\circ} \mathrm{F}\left(2^{\circ} \mathrm{C}\right.$ and $\left.\left.8^{\circ} \mathrm{C}\right)\right)$ : MMR vaccine, Hep A/Hep B/Hep A-Hep B/Hib/Hib-Hep B vaccines, HPV2/ HPV4 vaccines, influenza vaccine, polio vaccine, meningococcal-vaccines, pneumococcal vaccines, rotavirus vaccine, and the Diphtheria toxoid-, Tetanus toxoid-, and Pertussis-containing vaccines.

b) Before starting a vaccination program or opening a vaccination facility, develop and stick to a detailed written Routine Vaccine Storage and Handling Plan. Update plan annually and prepare in advance for emergency cases. Ensure that all staff members who administer or handle vaccines in any way are familiar with these plans. A designated primary vaccine coordinator and one alternate coordinator should be in charge of vaccine storage and handling of the vaccination facility.

c) Store vaccines in separate, self-contained units that refrigerate or freeze only. Never store any vaccines in a dormitory-style unit. If not possible, use it only for storage of our refrigerated vaccines. Maintain frozen vaccines in a separate stand-alone freezer.

d) Store vaccines in units with enough room to maintain the year's largest inventory without crowding.

e) It is very important to use only calibrated thermometers that have a Certificate of Traceability and Calibration Testing. Calibrate thermometers every 1 to 2 years from the last calibration testing date or according to the manufacturer's suggested timeline.

f) Have planned back-up storage unit(s) in the event of a power failure or other unforeseen event.

For more information on Vaccine Storage and handling go to http://www.cdc.gov/vaccines/recs/storage/ and CDC Vaccine Storage and Handling Toolkit.

\section{Vaccine administration ${ }^{8}$}

a) Before administering a vaccine, obtain the patient's complete immunization history at every visit. Additionally, screen the patient for contraindications and precautions before vaccine administration. Educate the patient on possible side effects of the vaccine before administering.

b) Be sure to practice hand hygiene before and after vaccine administration as well as when hands are soiled. Even though gloves are not mandatory for vaccine administration, wear them if you are likely to contact potentially infectious bodily fluids or if you have open lesions on your hands. If gloves are worn, change and perform hand hygiene between patients.

c) Inspect vaccine vial and/or diluents for contamination, expiration date, discoloration and precipitation before use.

d) Reconstitute the vaccine according to the manufacturing guidelines only at the time of administration, not few minutes before. Do NOT predraw doses before they are needed. This is required in order to prevent unnecessary preparation and wastage of vaccines. e) Remove vial dust cover, wipe the top with alcohol, and draw syringe according to standard preparation guidelines. Singledose vials should be used only for a single dose and then discarded. Once a multi-dose vial is opened, the vial should be date and discarded within 28 days of use or according to the manufacturer's date for the opened vial.

f) Oral route: For rotavirus vaccines, administer liquid slowly down one side of the inside cheek (between the cheek and gum) toward the back. Repetition if the patient vomits or gags is unnecessary.

g) Intranasal route: Patient should sit in an upright position with the head tilted slightly backwards. Insert nasal spray slightly into the nose and spray once to each nostril. Repetition is unnecessary even if the patient sneezes.

h) Subcutaneous route: First, pinch up some fatty tissue and insert the needle at a $45^{\circ}$ angle at the upper outer triceps of the arm or thigh for infants $<12$ months old and the upper outer triceps of the arm for persons 1 year or older. Inject the vaccine.

i) Intramuscular route: First, spread the skin of the site between the thumb and forefinger, isolating the deltoid muscle (upper arm) or anterolateral thigh muscle. Insert the needle fully at the muscle at a $90^{\circ}$ angle and inject the vaccine.

For more information on vaccine administration, go to http://www. cdc.gov/vaccines/pubs/pinkbook/downloads/vac-admin.pdf ${ }^{9}$

\section{Confidentiality and HIPAA ${ }^{3,4}$}

Immunization pharmacists need to know that all records sent to VAERS are part of a national, publicly available database, yet certain selected data in the reports are kept confidential as required by law. VAERS will not release personal information that identifies the vaccine recipient to the public. While HIPAA prohibits the release of protected health information without a patient's written authorization, this authorization is not required to release medical records "...to public health authorities for the purpose of preventing or controlling disease, injury, or disability" (HIPPA Privacy Rule). Pharmacists can report relevant info to VAERS.

\section{NC pharmacy laws and regulations ${ }^{10,11}$}

There are three requirements related to vaccine adverse events in NC. Pharmacists must:

a. Report all vaccine adverse events to the patient's primary physician.

b. Report all reportable vaccine adverse events to VAERS.

c. Provide evidence of documentation that the adverse event was reported to the patient's primary physician. Even though the $\mathrm{NC} \mathrm{BOP}$ regulations are silent on the documentation of adverse events, it still requires pharmacists to "maintain a record of any vaccine or immunization administered to the patient in a patient profile" (Pharmacy Laws of North Carolina). Hence, the adverse events after vaccine administration and notification to the patient's primary physician are required to be documented in the patient's profile.

In July 2014, the NC BOP is holding a public hearing on revisions to the immunization regulations. Adverse event reporting and documentations were not significantly affected by the proposed changes. 


\section{Conclusion}

Pharmacists have expanded their public health role by providing immunization services to adults and pediatric patients. With that expanded role is the duty to monitor for and report vaccine adverse events to the national vaccine reporting program and to the patient's primary care physician. These monitoring and reporting activities reduce the overall risk of harm and increase public awareness of vaccine safety. It is important to handle and store vaccine properly to increase their maximum usage and prevent wastage. Pharmacists can report these events while remaining fully compliant with HIPPA and confidentiality provisions of the federal and state laws.

\section{Acknowledgements}

None.

\section{Conflict of interest}

The author declares no conflict of interest.

\section{References}

1. http://www.cdc.gov/vaccinesafety/activities/vaers.html

2. http://www.fda.gov/BiologicsBloodVaccines/SafetyAvailability/ReportaProblem/VaccineAdverseEvents/QuestionsabouttheVaccineAdverseEventReportingSystemVAERS/

3. https://vaers.hhs.gov/index

4. https://vaers.hhs.gov/resources/vaersmaterialspublications

5. http://wonder.cdc.gov/wonder/help/vaers/reportable.htm

6. https://vaers.hhs.gov/esub/index

7. http://www.cdc.gov/vaccines/recs/storage/

8. CDC. Vaccine Administration. USA: CDC; 2015

9. http://www.cdc.gov/vaccines/pubs/pinkbook/downloads/vac-admin. pdf

10. http://www.ncbop.org/LawsRules/Statutes.pdf

11. http://www.ncbop.org/LawsRules/Rules.pdf 